\title{
Low-Knudsen-number photophoresis of aerosol spheroids
}

\author{
Chang L. Ou, Huan J. Keh* \\ Department of Chemical Engineering, National Taiwan University, Taipei 106-17, Taiwan, Republic of China
}

Received 21 April 2004; accepted 13 August 2004

\begin{abstract}
The photophoretic motion of a freely suspended aerosol spheroid exposed to a radiative heat flux that is oriented arbitrarily with respect to its axis of revolution is analytically studied. The Knudsen number is assumed to be so small that the fluid flow can be described by a continuum model with a thermal slip at the particle surface. In the limit of small Peclet and Reynolds numbers, the appropriate energy and momentum equations are solved using the bifocal-coordinate transformations. Expressions for the photophoretic velocity and force are obtained in closed form for various cases of prolate and oblate spheroidal particles. The average photophoretic velocity and force for an ensemble of identical, noninteracting spheroids with random orientation distribution are also determined. The results indicate that the aspect ratio and relative thermal conductivity of a spheroidal particle and its orientation with respect to the incident light can have significant effects on its photophoretic behavior.
\end{abstract}

(C) 2004 Elsevier Inc. All rights reserved.

Keywords: Photophoresis; Aerosol spheroids; Continuum regime; Thermal slip

\section{Introduction}

A small particle will migrate when it is suspended in a gaseous medium and exposed to an intense light beam. This phenomenon, which is a direct result of the uneven heating of the light-absorbing particle (and therefore of its adjacent gas molecules), was termed photophoresis by its discoverer, Ehrenhaft, around 1910 [1,2]. The photophoretic (or thermophoretic) effect can be explained in part by appealing to the kinetic theory of gases [3,4]. The higher energy molecules in the hot region of the gas impinge on the particle with greater momentum than molecules coming from the cold region, thereby leading to the migration of the particle in the direction opposite to the surface temperature gradient. Thus, the photophoretic force on an aerosol particle can be directed either toward (negative photophoresis) or away from (positive photophoresis) the light source, depending upon the optical characteristics of the particle. If the particle is opaque and the incident light energy is absorbed and

\footnotetext{
* Corresponding author.

E-mail address: huan@ntu.edu.tw (H.J. Keh).
}

dissipated directly at the front surface of the particle, positive photophoresis results. Conversely, if the light beam is partially transmitted and focused on the rear side of the particle, negative photophoresis may occur.

Photophoresis has been observed for many particulate materials in the diameter range between $10^{-8}$ and $10^{-3} \mathrm{~m}$, and at pressures from above 1 atm down to below 1 Torr, under illumination intensities comparable with sunlight [2]. Therefore, the results of photophoresis investigations are of interest to a wide variety of fields including cloud physics, aerosol science, and environmental engineering. For example, measurements of the photophoretic force or the reversal point from positive to negative photophoresis with the elaboration of photophoretic spectroscopy can be used to determine the physical properties, such as the complex refractive index, and the chemical composition of aerosol particles [5]. Photophoretic phenomena in aerosol particles subjected to coherent light beams have been applied to the development of laser atmospheric monitoring methods [6]. It was found that, due to the effect of both positive and negative photophoresis, some stratospheric aerosol particles may be caused to rise against gravity while others are induced to 
fall considerably more rapidly than they would under gravity alone [7]. Considering that radiative heat transfer can account for around $95 \%$ of the total heat flux in pulverized-coal furnaces, the driving force for photophoresis of small particles in combustion environments can be significantly greater than that for thermophoresis [8].

Assuming a small Knudsen number $(l / a$, where $a$ is the radius of the particle and $l$ is the mean free path of the surrounding gas molecules), a small Reynolds number, and a small Peclet number, as well as allowing the effects of temperature jump, thermal slip (thermoosmosis), and frictional slip at the gas-particle interface, Reed [9] and Mackowski [8] obtained the photophoretic velocity of a suspended aerosol sphere illuminated by an intense light beam as

$$
\mathbf{U}=-\frac{2 C_{s} \eta J}{3\left(1+2 C_{m} l / a\right)\left(2 k+k_{p}+2 k_{p} C_{t} l / a\right) \rho T_{0}} \mathbf{I} .
$$

Here, $\mathbf{I}$ is the incoming illumination energy flux (intensity of the incident light); $\rho, \eta$, and $k$ are the density, viscosity, and thermal conductivity, respectively, of the gas; $k_{p}$ is the thermal conductivity of the particle; $T_{0}$ is the absolute temperature of the bulk gas; $J$ is the so-called photophoretic asymmetry factor [10] defined by a special case of Eq. (33) or (34), which can be either positive (negative photophoresis) or negative (positive photophoresis); and $C_{S}$, $C_{t}$, and $C_{m}$ are dimensionless coefficients accounting for the thermal slip, temperature jump, and frictional slip phenomena, respectively, at the particle surface and must be determined experimentally for each gas-solid system. A set of kinetic-theory values for complete thermal and momentum accommodations appear to be $C_{s}=1.17, C_{t}=2.18$, and $C_{m}=1.14$ [11]. Recently, kinetic-theory values of these slip coefficients have been obtained accurately under various conditions [12,13]. According to Eq. (1), the photophoretic velocity at illumination of an intensity comparable with the solar constant $\left(1353 \mathrm{~W} \mathrm{~m}^{-2}\right)$ is of the order of $10^{-5} \mathrm{~m} \mathrm{~s}^{-1}$.

When the Knudsen number is vanishingly small, the effects of temperature jump and frictional slip at the particle surface disappear and Eq. (1) reduces to its limit,

$\mathbf{U}=-\frac{2 C_{s} \eta J}{3\left(2 k+k_{p}\right) \rho T_{0}} \mathbf{I}$.

The photophoretic velocity predicted by Eq. (2) for an aerosol sphere, which is independent of particle size, is in fair agreement with the experimental result obtained in the continuum regime with constant illumination intensity [9].

Most aerosol particles and their aggregates are not spherical $[14,15]$ and it is therefore of considerable interest to examine the effect of particle shape on photophoresis. The photophoretic theories of nonspherical particles differ from those of spheres in that there is an orientational problem. In general, the photophoretic mobility of a nonspherical particle is anisotropic and the velocity of the particle is no longer collinear with the incident light beam. The techniques for calculating photophoretic forces and torques have been discussed to some extent for general nonspherical particles in the free molecule regime [16-18] and in the continuum regime $[18,19]$. Recently, the photophoretic motion of an infinitely long circular cylinder in the direction normal to its axis has been studied with consideration of the effects of temperature jump, thermal slip, and frictional slip at the particle surface and an analytical expression for the migration velocity of the particle is obtained [20]. The result indicates that the photophoretic mobility of a cylindrical particle can be greater or smaller than that of a sphere, depending on the ratio of particle to gas thermal conductivity and the relative magnitude of the temperature jump or frictional slip coefficient at the particle surface.

In this paper we present an analysis of the photophoretic motion of an aerosol spheroid with a thermal slip at the particle surface. The spheroidal particle can be either prolate or oblate and is oriented arbitrarily with respect to the imposed light beam. Analytical formulas for the photophoretic velocities and forces of a single spheroid and of an ensemble of identical spheroids as functions of relevant parameters are obtained in closed forms.

\section{Analysis}

Consider the photophoretic motion of a spheroidal particle suspended in an unbounded gaseous medium, as shown in Fig. 1. For convenience, the rectangular coordinate system $(x, y, z)$ is established with its origin located at the particle center so that the surface of the spheroid $\left(S_{p}\right)$ is represented by the equation

$\frac{z^{2}}{a^{2}}+\frac{x^{2}+y^{2}}{b^{2}}=1$

where $a$ and $b$ are the half-length along the axis of revolution and the maximum cross-sectional radius, respectively, of the spheroid. The radiative heat flux of the incident light, which is oriented arbitrarily with respect to the particle's axis of revolution, can be taken as a combination of the longitudinal component $I_{1}$ and the transversal component $I_{2}$, both

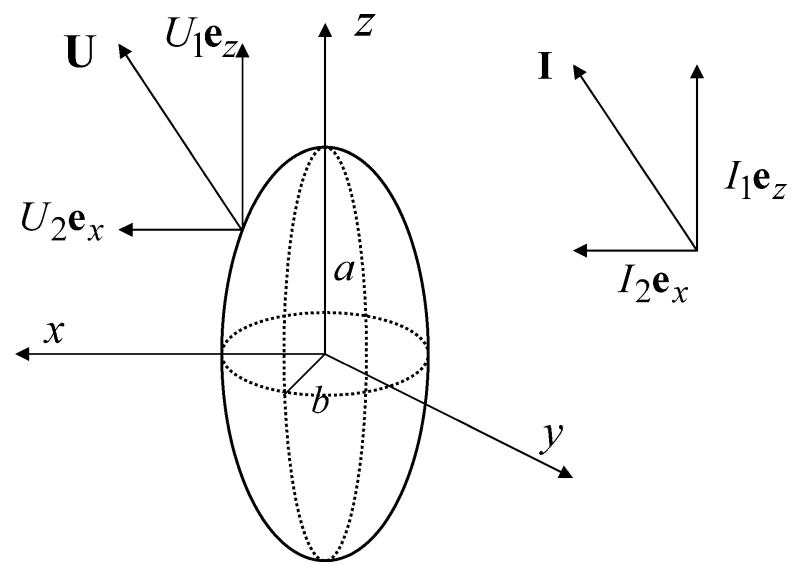

Fig. 1. Geometrical sketch for the photophoretic motion of a spheroidal particle. 
are positive, and equal to $I_{1} \mathbf{e}_{z}+I_{2} \mathbf{e}_{x}$, where $\mathbf{e}_{x}$ and $\mathbf{e}_{z}$ are the unit vectors in the $x$ and $z$ directions, respectively. It is assumed that the Knudsen number is so small (say, less than 0.1) that the fluid flow is in the continuum regime, and the Knudsen layer at the particle surface is thin in comparison with $a$ and $b$. The larger one between $a$ and $b$ is intuitively more relevant to the transport of physical quantities involved and might be an appropriate characteristic length to define the Knudsen and other dimensionless numbers. In the continuum-regime model, the gas-kinetic photophoretic or thermophoretic force is taken to arise from a thermal slip along the particle surface due to the existence of a tangential temperature gradient at the particle-fluid interface [3]. Our purpose here is to determine the photophoretic velocity of the spheroidal particle. First, it is necessary to ascertain the temperature distribution inside and outside the particle.

\subsection{Temperature distribution}

The problem of photophoresis of a spheroidal particle is considered for the case where the Peclet and Reynolds numbers are small (say, both are less than 0.1, such that the effects of convections of heat and momentum are neglected). The energy equation governing the temperature distribution $T(\mathbf{r})$ for the fluid phase of constant thermal conductivity $k$ is Laplace's equation,

$\nabla^{2} T=0$,

where $\mathbf{r}$ is the position vector from the particle center. The temperature field $T_{p}(\mathbf{r})$ within the spheroid can be expressed as the superposition

$T_{p}=T_{0}+T_{1}+T_{2}$,

where $T_{0}$ is the temperature of the isothermal bulk gas; $T_{1}(\mathbf{r})$ and $T_{2}(\mathbf{r})$ represent the disturbances produced by the longitudinal and transversal components, respectively, of the incident light. The temperature disturbances inside the radiationabsorbing particle are described by

$\nabla^{2} T_{i}=-\frac{1}{k_{p}} q_{i}, \quad i=1,2$,

where $k_{p}$ is the thermal conductivity of the particle, which is assumed to be independent of temperature, $q_{i}(\mathbf{r})$ are the volumetric thermal energy generation rates resulting from local radiation absorption, and the subscripts 1 and 2 denote the effects caused by the longitudinal and transversal components, respectively, of the incident light.

For a plane monochromatic wave, the source functions $q_{i}(\mathbf{r})$ are related to the electric field inside the particle according to the Lorenz-Mie theory [8,21],

$q_{1}=\frac{4 \pi \nu \kappa}{\lambda} I_{1} B_{1}(\tau, \omega)$,
$q_{2}=\frac{4 \pi \nu \kappa}{\lambda} I_{2} B_{2}(\tau, \omega, \phi)$.
Here, $v$ and $\kappa$ are the real and imaginary parts of the complex refractive index $\bar{N}(\bar{N}=v+i \kappa)$ of the particle, $\lambda$ is the wavelength of the incident radiation, $B_{1}(\tau, \omega)$ and $B_{2}(\tau, \omega, \phi)$ are the dimensionless electric field distribution functions, and $\tau, \omega$, and $\phi$ are the variables related to the bifocal-coordinate transformations. For prolate spheroids $(b<a)$, the coordinate transformation used is

$x=c \sinh \xi \sin \varphi \cos \phi$,

$y=c \sinh \xi \sin \varphi \sin \phi$,

$z=c \cosh \xi \cos \varphi$,

$\tau=\cosh \xi, \quad \omega=\cos \varphi$,

$a=c \cosh \xi_{0}, \quad b=c \sinh \xi_{0}$,

$c=\left(a^{2}-b^{2}\right)^{1 / 2}$,

while for oblate spheroids $(b>a)$, the coordinate transformation used is

$x=c \cosh \xi \sin \varphi \cos \phi$,

$y=c \cosh \xi \sin \varphi \sin \phi$,

$z=c \sinh \xi \cos \varphi$,

$\tau=i \sinh \xi, \quad \omega=\cos \varphi$,

$a=c \sinh \xi_{0}, \quad b=c \cosh \xi_{0}$,

$c=\left(b^{2}-a^{2}\right)^{1 / 2}$.

Here, we employ the right-handed bifocal coordinate system of revolution $(\xi, \varphi, \phi)$ with the origin (midpoint between the foci) at the center of the spheroid, and $i=\sqrt{-1}$. These coordinates range over the values $0 \leqslant \xi<\infty, 0 \leqslant \varphi \leqslant \pi$, $0 \leqslant \phi<2 \pi$. The coordinate surface $\xi=\xi_{0}$ (or $\tau=\tau_{0}$ ) corresponds to the surface of the spheroid.

The boundary conditions require that (i) the normal heat fluxes be continuous at the interface between the particle and the fluid phase, (ii) the temperatures be continuous at the particle surface when the effect of temperature jump is negligible (valid for the case of small Knudsen numbers), (iii) the temperature be finite inside the particle, and (iv) the temperature field far from the particle approach the prescribed bulk value. Thus,

$$
\begin{aligned}
\text { on } S_{p}: & \mathbf{n} \cdot k \nabla T=\mathbf{n} \cdot k_{p} \nabla T_{p}, \\
& T=T_{p},
\end{aligned}
$$

inside $S_{p}: \quad T_{p}$ is finite,

as $|\mathbf{r}| \rightarrow \infty: \quad T \rightarrow T_{0}$,

where $\mathbf{n}$ is the unit vector outwardly normal to the particle surface $S_{p}$. In Eq. (11), the flux due to radiation heat transfer, which was taken into account by Akhtaruzzaman and Lin [22] in studying the photophoresis of a spherical particle in the free molecule regime (in a rarefied gas with large Knudsen number), is neglected with the assumption that the surface temperature of the particle is not very high. 
A general solution of Eq. (4) that satisfies boundary condition (14) is [23]

$$
\begin{aligned}
T= & T_{0}+\frac{I_{1} c}{k} \sum_{n=0}^{\infty} C_{1 n} P_{n}^{0}(\omega) Q_{n}^{0}(\tau) \\
& +\frac{I_{2} c}{k} \sum_{n=1}^{\infty} C_{2 n} P_{n}^{1}(\omega) Q_{n}^{1}(\tau) \cos \phi,
\end{aligned}
$$

and a general solution of Eqs. (5)-(8) that satisfies boundary condition (13) is

$$
\begin{aligned}
T_{p}= & T_{0}+\frac{I_{1} c}{k_{p}} \sum_{n=0}^{\infty} P_{n}^{0}(\omega)\left[D_{1 n} P_{n}^{0}(\tau)+\frac{R_{1 n}(\tau)}{\int_{-1}^{1}\left[P_{n}^{0}(\omega)\right]^{2} \mathrm{~d} \omega}\right] \\
& +\frac{I_{2} c}{k_{p}} \sum_{n=1}^{\infty} P_{n}^{1}(\omega) \\
& \times\left[D_{2 n} P_{n}^{1}(\tau)+\frac{R_{2 n}(\tau)}{\int_{-1}^{1}\left[P_{n}^{1}(\omega)\right]^{2} \mathrm{~d} \omega}\right] \cos \phi
\end{aligned}
$$

where

$$
\begin{aligned}
& R_{\text {in }}(\tau)=-P_{n}^{i-1}(\tau) \int_{\tau}^{\tau_{0}} A_{\text {in }}(t) Q_{n}^{i-1}(t) h_{\text {in }}(t) \mathrm{d} t \\
& \quad+Q_{n}^{i-1}(\tau) \int_{0}^{\tau} A_{\text {in }}(t) P_{n}^{i-1}(t) h_{\text {in }}(t) \mathrm{d} t, \quad i=1,2 .
\end{aligned}
$$

In the above equations,

$$
\begin{aligned}
A_{\text {in }}(t)= & \left(t^{2}-1\right)^{-1} \\
& \times\left[\overline{P_{n}^{i-1}}(t) Q_{n}^{i-1}(t)-P_{n}^{i-1}(t) \overline{Q_{n}^{i-1}}(t)\right]^{-1}, \\
h_{1 n}(t)= & \frac{4 \pi v \kappa c}{\lambda} \int_{-1}^{1}\left(t^{2}-\omega^{2}\right) B_{1}(t, \omega) P_{n}^{0}(\omega) \mathrm{d} \omega, \\
h_{2 n}(t)= & \frac{4 v \kappa c}{\lambda} \int_{0}^{2 \pi} \int_{-1}^{1}\left(t^{2}-\omega^{2}\right) \\
& \times B_{2}(t, \omega, \phi) P_{n}^{1}(\omega) \cos \phi \mathrm{d} \omega \mathrm{d} \phi,
\end{aligned}
$$

$P_{n}^{m}$ and $Q_{n}^{m}$ are the associated Legendre functions of the first and second kinds, respectively, of order $m$ and degree $n$, $C_{1 n}, D_{1 n}, C_{2 n}$, and $D_{2 n}$ are the unknown coefficients to be determined using boundary conditions (11) and (12), and the overbars on $P_{n}^{m}$ and $Q_{n}^{m}$ mean differentiation with respect to their arguments. The derivation of solution (16) is presented in Appendix A.

Applying Eqs. (11) and (12) to Eqs. (15) and (16), we obtain the unknown coefficients as

$$
\begin{aligned}
C_{i n}= & \frac{k^{*}}{\overline{Q_{n}^{i-1}}\left(\tau_{0}\right)}\left[R_{i n}\left(\tau_{0}\right)-\frac{P_{n}^{i-1}\left(\tau_{0}\right)}{\overline{P_{n}^{i-1}}\left(\tau_{0}\right)} \overline{R_{\text {in }}}\left(\tau_{0}\right)\right] \\
& \times\left[k^{*} \frac{Q_{n}^{i-1}\left(\tau_{0}\right)}{\overline{Q_{n}^{i-1}}\left(\tau_{0}\right)}-\frac{P_{n}^{i-1}\left(\tau_{0}\right)}{\overline{P_{n}^{i-1}}\left(\tau_{0}\right)}\right]^{-1},
\end{aligned}
$$

$$
\begin{aligned}
D_{i n}= & {\left[R_{i n}\left(\tau_{0}\right)-k^{*} \frac{Q_{n}^{i-1}\left(\tau_{0}\right)}{\overline{Q_{n}^{i-1}}\left(\tau_{0}\right)} \overline{R_{i n}}\left(\tau_{0}\right)\right] } \\
& \times\left[k^{*} \overline{P_{n}^{i-1}}\left(\tau_{0}\right) \frac{Q_{n}^{i-1}\left(\tau_{0}\right)}{\overline{Q_{n}^{i-1}}\left(\tau_{0}\right)}-P_{n}^{i-1}\left(\tau_{0}\right)\right]^{-1},
\end{aligned}
$$

where $k^{*}=k_{p} / k, \tau_{0}=\left[1-(b / a)^{2}\right]^{-1 / 2}$, which equals $\tau$ with $\xi=\xi_{0}$, and the overbar on $R_{i n}(\tau)$ means differentiation with respect to $\tau$. Evidently, $b / a<1$ and $\tau_{0} \geqslant 1$ for a prolate spheroid; $b / a>1$ and $\tau_{0}^{2} \leqslant 0$ for an oblate spheroid. Since the thermal conductivity of an aerosol particle is usually greater than that of the surrounding gas, the value of the relative conductivity $k^{*}$ will exceed unity under most practical circumstances. Note that, if the effect of temperature jump is included at the particle surface to replace the boundary condition given by Eq. (12), the solved coefficients $C_{i n}$ and $D_{\text {in }}$ become dependent on the angular variable $\omega$ and an analytical solution for the temperature distribution is not feasible for bifocal coordinate systems [24,25].

\subsection{Photophoretic velocity}

With knowledge of the solution for the temperature field, we can now proceed to find the photophoretic velocity of the particle. Due to the low Reynolds numbers encountered in the photophoresis, the fluid flow is governed by the Stokes equations,

$\eta \nabla^{2} \mathbf{v}-\nabla p=\mathbf{0}$,

$\nabla \cdot \mathbf{v}=0$

where $\mathbf{v}(\mathbf{r})$ is the fluid velocity and $p(\mathbf{r})$ is the dynamic pressure.

Owing to the thermal slip velocity along the particle surface (or more precisely, the outer edge of the thin Knudsen layer), first predicted by Maxwell [3], and the fluid at rest far from the particle, the boundary conditions for the fluid velocity are

on $S_{p}^{+}: \quad \mathbf{v}=\mathbf{U}+\mathbf{v}_{s}$,

as $|\mathbf{r}| \rightarrow \infty: \quad \mathbf{v}=\mathbf{0}$,

where

$\mathbf{v}_{s}=C_{s} \frac{\eta}{\rho T_{0}} \nabla_{s} T$,

and $\mathbf{U}$ is the instantaneous photophoretic velocity of the particle to be determined. The validity of the expression for the thermal slip velocity in Eq. (27) is based on the assumption that the fluid is only slightly nonuniform in temperature on the length scale of the particle size, and the tangential temperature gradient $\nabla_{S} T$ can be obtained from Eqs. (15) and (21). The contribution from the effect of frictional slip at the particle surface is neglected in Eq. (25) since it is small compared to thermal slip for the case of small Knudsen numbers. If frictional slip is included, an analytical solution for the fluid velocity distribution or particle velocity is not feasible for bifocal coordinate systems $[24,25]$, similar to the 
case of thermal field with temperature jump at the particle surface.

For the photophoretic motion of a spheroidal particle (having three mutually perpendicular planes of symmetry) exposed to a uniform light beam in the creeping-flow continuum regime, there is no rotation or preferred orientation of the particle due to the linearity of the governing equations (4), (6), (23), and (24) as well as the axial and fore/aft symmetry of the particle shape [26,27]. Even so, the problem of solving Eqs. (23) and (24) with the boundary conditions (25) and (26) may not be a simple one for the migration of a spheroid. Fortunately, the use of the constraint of zero hydrodynamic force on the particle and the Lorentz reciprocal theorem for the Stokes flow [26] gives the surface-integral formula for the migration velocity of a spheroidal particle [28],

$\mathbf{U}=-\frac{1}{3 V_{p}} \iint_{S_{p}^{+}}(\mathbf{n} \cdot \mathbf{r}) \mathbf{v}_{s} \mathrm{~d} S$,

where $V_{p}$ is the volume of the spheroid.

Substitution of Eq. (15) into Eq. (27) leads to the slip velocity at the particle surface,

$$
\begin{aligned}
\mathbf{v}_{s}= & \mathbf{e}_{\varphi} \frac{C_{s} \eta}{\rho T_{0}} I_{1}\left(\frac{1-\omega^{2}}{\tau_{0}^{2}-\omega^{2}}\right)^{1 / 2} \sum_{n=0}^{\infty} C_{1 n} Q_{n}^{0}\left(\tau_{0}\right) \overline{P_{n}^{0}}(\omega) \\
& +\frac{C_{s} \eta}{\rho T_{0}} I_{2}\left[\mathbf{e}_{\varphi} \cos \phi\left(\frac{1-\omega^{2}}{\tau_{0}^{2}-\omega^{2}}\right)^{1 / 2}\right. \\
& \times \sum_{n=1}^{\infty} C_{2 n} Q_{n}^{1}\left(\tau_{0}\right) \overline{P_{n}^{1}}(\omega) \\
& +\mathbf{e}_{\phi} \sin \phi\left(\tau_{0}^{2}-1\right)^{-1 / 2}\left(1-\omega^{2}\right)^{-1 / 2} \\
& \left.\times \sum_{n=1}^{\infty} C_{2 n} Q_{n}^{1}\left(\tau_{0}\right) P_{n}^{1}(\omega)\right],
\end{aligned}
$$

where $\mathbf{e}_{\varphi}$ and $\mathbf{e}_{\phi}$ are unit vectors in the bifocal coordinate system. When $\mathbf{v}_{s}$ in Eq. (28) is replaced by the form of Eq. (29) with Eqs. (17)-(21) and integrating over the particle surface, we obtain the migration velocity of a general spheroid undergoing photophoresis under an incident light of arbitrary direction as

$\mathbf{U}=U_{1} \mathbf{e}_{z}+U_{2} \mathbf{e}_{x}$,

where the longitudinal and transversal components are

$U_{1}=-\frac{C_{s} \eta}{k \rho T_{0}} I_{1} J_{1} \alpha\left(1+\frac{\alpha}{\beta} k^{*}\right)^{-1}$

$U_{2}=-\frac{1}{2} \frac{C_{s} \eta}{k \rho T_{0}} I_{2} J_{2} \beta\left(1+\frac{\beta}{\alpha+1} k^{*}\right)^{-1}$.

In Eqs. (31) and (32), $J_{1}$ and $J_{2}$ are the so-called photophoretic asymmetry factors,

$J_{1}=\frac{6 \pi \nu \kappa c}{\lambda \tau_{0}}\left[Q_{1}^{0}\left(\tau_{0}\right)-\frac{P_{1}^{0}\left(\tau_{0}\right)}{\overline{P_{1}^{0}}\left(\tau_{0}\right)} \overline{Q_{1}^{0}}\left(\tau_{0}\right)\right]$

$$
\begin{aligned}
& \times \int_{0}^{\tau_{0}} \int_{-1}^{1} A_{1 n}(t) P_{1}^{0}(t)\left(t^{2}-\omega^{2}\right) B_{1}(t, \omega) \mathrm{d} \omega \mathrm{d} t, \\
J_{2}= & \frac{3 v \kappa c}{\lambda \tau_{0}}\left[Q_{1}^{1}\left(\tau_{0}\right)-\frac{P_{1}^{1}\left(\tau_{0}\right)}{\overline{P_{1}^{1}}\left(\tau_{0}\right)} \overline{Q_{1}^{1}}\left(\tau_{0}\right)\right] \\
& \times \int_{0}^{\tau_{0}} \int_{0}^{2 \pi} \int_{-1}^{1} A_{2 n}(t) P_{1}^{1}(t)\left(t^{2}-\omega^{2}\right) \\
& \times B_{2}(t, \omega, \phi) \cos \phi \mathrm{d} \omega \mathrm{d} \phi \mathrm{d} t,
\end{aligned}
$$

and $\alpha$ and $\beta$ are positive values related to $\tau_{0}$ or the aspect ratio $a / b$,

$\alpha=\left(\tau_{0}^{2}-1\right)\left(\tau_{0} \operatorname{coth}^{-1} \tau_{0}-1\right)$,

$\beta=\tau_{0}\left[\tau_{0}-\left(\tau_{0}^{2}-1\right) \operatorname{coth}^{-1} \tau_{0}\right]$.

In general, the direction of the nonspherical particle migration will not be parallel to the incident light.

The photophoretic asymmetry factors $J_{1}$ and $J_{2}$ given by Eqs. (33) and (34) depend on the complex refractive index $(\bar{N}=\nu+i \kappa)$ and the normalized size $(2 \pi c / \lambda)$ of the particle. Each of them represents a weighted integration of the heat source function over the particle volume and defines the sign and magnitude of the photophoretic velocity. For a completely opaque spheroidal particle, the heat sources are concentrated on the illuminated part of the particle surface; namely, the functions $B_{1}$ and $B_{2}$ in Eqs. (7) and (8) are given by

$$
\begin{aligned}
& B_{1}(t, \omega)= \begin{cases}-\frac{\lambda}{2 \pi \nu \kappa c} \delta\left(t-\tau_{0}\right) \cos \varphi & \text { for } \frac{\pi}{2} \leqslant \varphi \leqslant \pi, \\
0 & \text { for } 0 \leqslant \varphi \leqslant \frac{\pi}{2},\end{cases} \\
& B_{2}(t, \omega, \phi)= \begin{cases}-\frac{\lambda}{2 \pi \nu \kappa c} \delta\left(t-\tau_{0}\right) \sin \varphi \cos \phi & \text { for } \frac{\pi}{2} \leqslant \phi \leqslant \frac{3 \pi}{2}, \\
0 & \text { for } \frac{\pi}{2} \leqslant|\phi-\pi| \leqslant \pi,\end{cases}
\end{aligned}
$$

where $\delta\left(t-\tau_{0}\right)$ is a Dirac delta function which equals infinity if $t=\tau_{0}$ and vanishes otherwise.

The substitution of Eqs. (37) and (38) into Eqs. (33) and (34) results in

$$
\begin{aligned}
& J_{1}=-\frac{5 \tau_{0}^{2}-3}{10\left(\tau_{0}^{2}-1\right)}, \\
& J_{2}=-\frac{5 \tau_{0}^{2}-1}{10 \tau_{0}^{2}} .
\end{aligned}
$$

The factors $J_{1}$ and $J_{2}$ predicted by Eqs. (39) and (40) depend only on the shape of the spheroid, and their values are always negative, indicating the positive photophoresis as expected. Since the Knudsen number is assumed to be small, the normalized photophoretic velocities obtained in Eqs. (31) and (32) with Eqs. (39) and (40) do not depend directly on the linear dimensions of the spheroid but only on its aspect ratio $a / b$ and the thermal conductivity ratio $k^{*}$. 
If the particle is prevented from moving (i.e., $\mathbf{U}$ in boundary condition (25) is set equal to zero), the resulted hydrodynamic force exerted on the particle can be taken as the photophoretic force due to the incident light. This force can be expressed as

$\mathbf{F}=6 \pi \eta\left(R_{1} U_{1} \mathbf{e}_{z}+R_{2} U_{2} \mathbf{e}_{x}\right)$,

where analytical formulas for the radii of the "equivalent sphere," $R_{1}$ and $R_{2}$, are given by Happel and Brenner [26, pp. 222-224]. Note that the photophoretic force acting on the particle depends not only on the shape and relative orientation but also on the size of the particle.

Usually, experimental data on the photophoretic velocity are average results. It is understood that there is no rotation or preferred orientation of a spheroid in a uniform creeping flow. For an ensemble of identical, noninteracting spheroids with random orientation distribution, the average particle velocity (aligned with the direction of $\mathbf{I}$ ) can be evaluated from the longitudinal and transversal mobilities [26, p. 207],

$\mathbf{U}_{\mathrm{av}}=\frac{1}{3}\left(m_{1}+2 m_{2}\right) \mathbf{I}$,

where $m_{1}=U_{1} / I_{1}$ and $m_{2}=U_{2} / I_{2}$. Similarly, the average photophoretic force acting on the particles of this ensemble can be obtained using Eq. (41), with the result

$\mathbf{F}_{\mathrm{av}}=2 \pi \eta\left(R_{1} m_{1}+2 R_{2} m_{2}\right) \mathbf{I}$.

The photophoretic mobilities $m_{1}$ and $m_{2}$ in Eqs. (42) and (43) can be calculated using Eqs. (31) and (32). For the particular case of $k^{*}=0$ or a perfectly insulating particle (which is of little concern in aerosol physics), both $m_{1}$ and $m_{2}$ have relatively maximal magnitudes. The finite value of the thermal conductivity of the particle decreases the tangential temperature gradient at the particle surface and results in a reduction of the photophoretic mobility.

When $a / b=1$ (i.e., $\tau_{0}^{2} \rightarrow \pm \infty$ ), Eqs. (35)-(40) give $J_{1}=J_{2}=J=-1 / 2, \alpha=1 / 3$, and $\beta=2 / 3$, and both Eq. (31) and Eq. (32) become

$m_{1}=m_{2}=m_{s} \equiv \frac{C_{s} \eta}{6 k \rho T_{0}}\left(1+\frac{1}{2} k^{*}\right)^{-1}$,

which is the reduced result for the photophoretic mobility of a spherical particle given by Eq. (2). For this case, $R_{1}=$ $R_{2}=a=b$ (the radius of the particle) in Eq. (41) or (43), which leads to Stokes' law.

In the limiting case of $a / b \rightarrow 0$, Eqs. (31) and (32) with factors $J_{1}$ and $J_{2}$ given by Eqs. (39) and (40) can be asymptotically expressed as

$$
\begin{aligned}
m_{1}= & \frac{3 C_{s} \eta}{40 k \rho T_{0} k^{*}}\left\{2 \pi\left(\frac{a}{b}\right)^{2}+\left(\pi^{2}+4 k^{*}\right)\left(\frac{a}{b}\right)^{4}\right. \\
& \left.+O\left[\left(\frac{a}{b}\right)^{6}\right]\right\}
\end{aligned}
$$

$$
\begin{aligned}
m_{2}= & \frac{C_{s} \eta}{320 k \rho T_{0}}\left\{8 \pi\left(\frac{a}{b}\right)^{-2}+2\left(8+\pi^{2} k^{*}\right)\right. \\
& +\pi\left[16\left(6-k^{*}\right)+\pi^{2} k^{*}\left(1-k^{*}\right)\right]\left(\frac{a}{b}\right)^{2} \\
& \left.+O\left[\left(\frac{a}{b}\right)^{4}\right]\right\} .
\end{aligned}
$$

These are the results for the photophoretic mobility of a disklike structure of radius $b$. Clearly, $m_{1}=0$ (for $k^{*}>0$ ) and $m_{2} \rightarrow \infty$ as $a / b=0\left(\tau_{0}=0, \alpha=1, \beta=0, J_{1}=-3 / 10\right.$, and $J_{2} \rightarrow-\infty$ ). This is, a disk-shaped particle with its edge aligned with the incident light will have substantially greater photophoretic mobility than with its axis aligned with the incident light.

In the other limiting case of $a / b \rightarrow \infty$, Eqs. (31) and (32) together with Eqs. (39) and (40) reduce to

$$
\begin{aligned}
m_{1}= & \frac{C_{s} \eta}{10 k \rho T_{0}}\left[-\left(\frac{b}{a}\right)^{-2} \zeta-2 k^{*}\left(\frac{b}{a}\right)^{-2} \zeta^{2}-\frac{3}{4}\left(\frac{b}{a}\right)^{2}\right. \\
& \left.-\frac{1}{2} k^{* 2}\left(\frac{b}{a}\right)^{-2} \zeta^{3}+O\left(\zeta^{2}\right)\right], \\
m_{2}= & \frac{C_{s} \eta}{40 k \rho T_{0}}\left[\frac{8}{1+k^{*}}-\frac{4 k^{*}}{\left(1+k^{*}\right)^{2}} \zeta-\frac{2 k^{*}}{\left(1+k^{*}\right)^{3}} \zeta^{2}\right. \\
& \left.-\frac{3 k^{*}}{\left(1+k^{*}\right)^{2}}\left(\frac{b}{a}\right)^{4}+O\left(\zeta^{3}\right)\right]
\end{aligned}
$$

where

$\zeta=\left(\frac{b}{a}\right)^{2} \ln \frac{b}{2 a}$

implying that $\zeta=0$ as $b / a=0$. These mobility results correspond to the photophoresis of a needlelike structure of length $a$. Evidently, $m_{1} \rightarrow \infty$ and $m_{2}=C_{s} \eta / 5 k \rho T_{0}\left(1+k^{*}\right)$ as $b / a=0\left(\tau_{0}=1, \alpha=0, \beta=1, J_{1} \rightarrow-\infty\right.$, and $J_{2}=$ $-2 / 5)$. That is, a needle-shaped particle aligned with the incident light will have much higher photophoretic mobility than one oriented normal to the light beam.

Note that both the photophoretic mobilities $m_{2}$ at $a / b=0$ and $m_{1}$ at $b / a=0$ approach infinity, analogously to the corresponding translational mobilities of a spheroid driven to move by a body force field [26]. Also, the value of $m_{2}$ at $b / a=0$ is slightly smaller than that of the photophoretic mobility of an infinitely long circular cylinder in the direction normal to its axis, which equals $C_{s} \eta / 4 k \rho T_{0}\left(1+k^{*}\right)$ [20].

\section{Results and discussion}

The numerical results for the longitudinal mobility $m_{1}$ and transversal mobility $m_{2}$, given by Eqs. (31) and (32), with the asymmetry factors $J_{1}$ and $J_{2}$, given by Eqs. (39) 


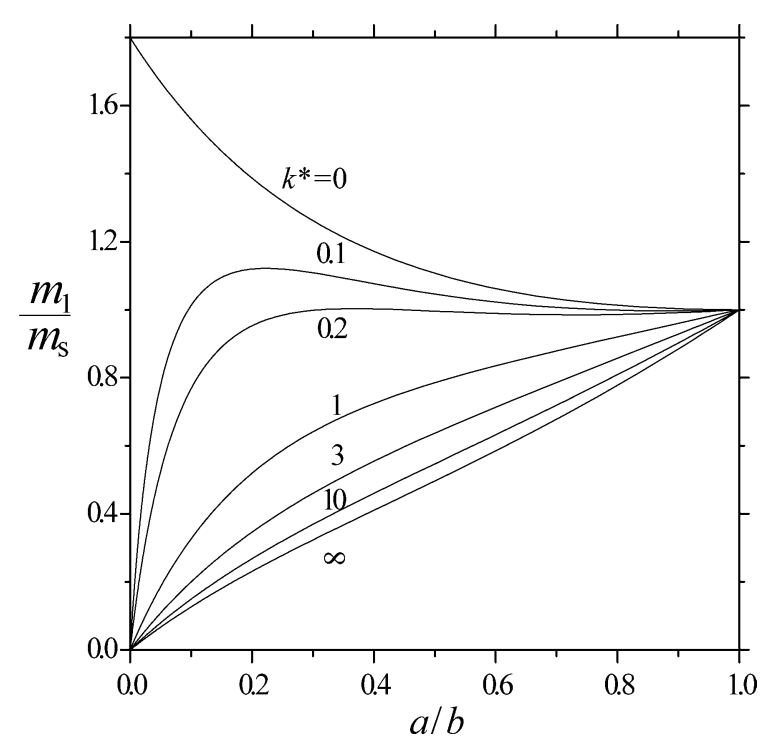

(a)

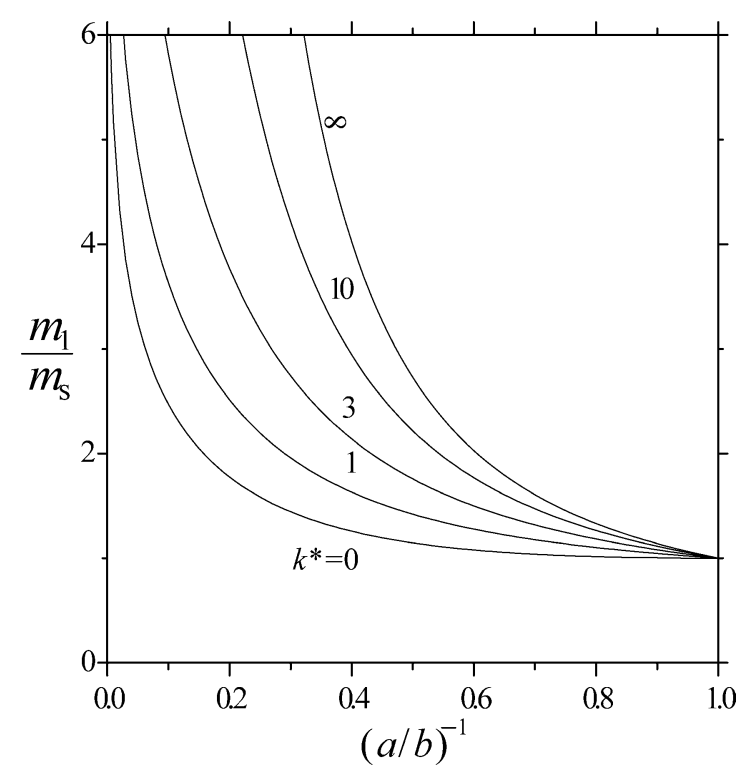

(b)

Fig. 2. Plots of the normalized photophoretic mobility of a spheroidal particle in the longitudinal direction versus the aspect ratio for various values of the relative thermal conductivity of the particle: (a) oblate spheroid; (b) prolate spheroid.

and (40), for a photophoretic spheroid as functions of the aspect ratio $a / b$ are plotted in Figs. 2 and 3, respectively, for various values of the thermal conductivity ratio $k^{*}$ over the entire ranges. The magnitudes of these mobilities are normalized by the corresponding photophoretic mobility $m_{s}$ of a spherical particle given by Eq. (44). On the other hand, the normalized photophoretic mobilities $m_{1} / m_{s}$ and $m_{2} / m_{s}$ of a spheroidal particle versus the conductivity ratio $k^{*}$ for several different values of $a / b$ are plotted in Figs. 4 and 5, respectively. The cases of $k^{*}<1$, which is not likely to exist in practice, is considered here for the sake of numerical comparison.

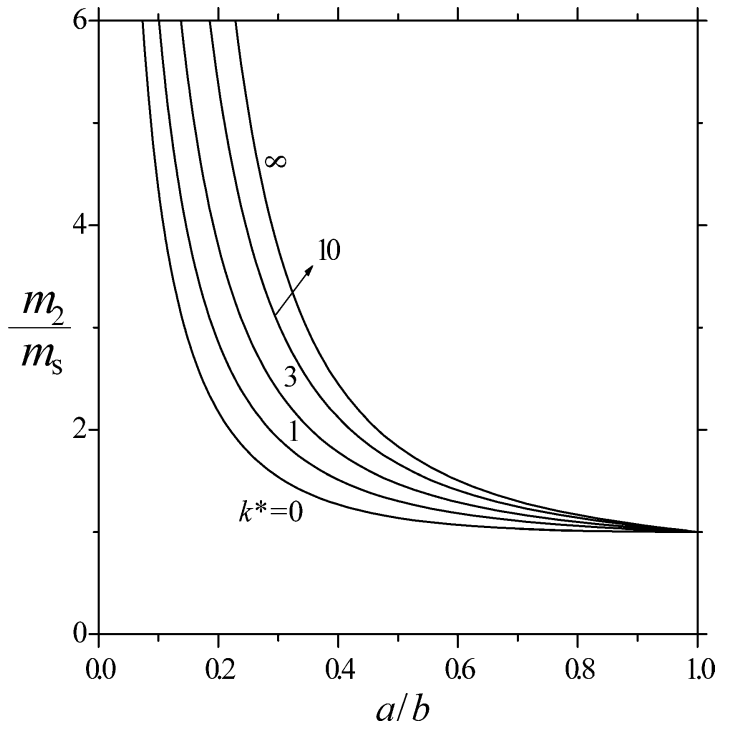

(a)

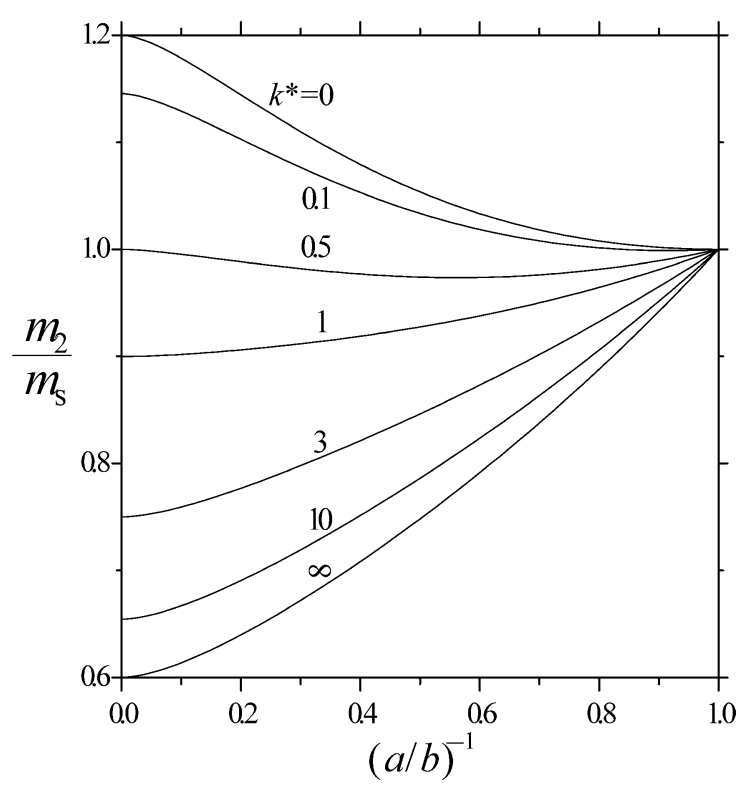

(b)

Fig. 3. Plots of the normalized photophoretic mobility of a spheroidal particle in the transverse direction versus the aspect ratio for various values of the relative thermal conductivity of the particle: (a) oblate spheroid; (b) prolate spheroid.

It can be seen in Figs. 2 and 4 that the normalized longitudinal photophoretic mobility $m_{1} / m_{s}$ of a spheroid increases monotonically with an increase in its aspect ratio $a / b$ in the whole range of $0 \leqslant a / b<\infty$ for a specified practical value of $k^{*}$ (say, $k^{*} \geqslant 1$ ). This behavior is understandable since the fraction of the thermal slip of the fluid at the particle surface in the axial direction, which drives the movement of the particle, increases with the increase of $a / b$. Therefore, the spherical approximation underestimates the longitudinal photophoretic mobility for prolate spheroids and overestimates it for oblate ones. For the particular case of $a / b=0$ (but $k^{*} \neq 0$ ), $m_{1} / m_{s}=0$, which is consistent with Eq. (45). This result is understandable since a disk of zero 


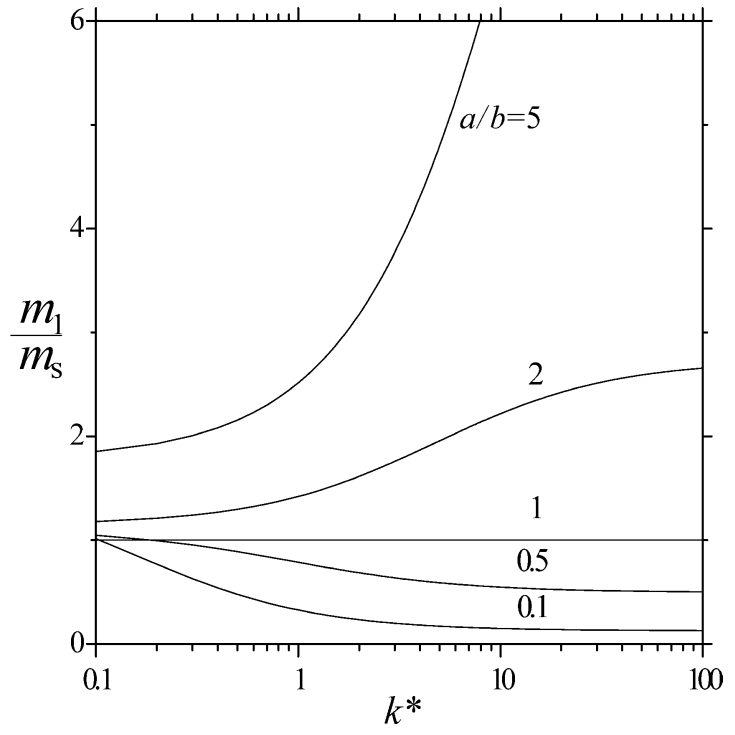

Fig. 4. Plots of the normalized photophoretic mobility of a spheroidal particle in the longitudinal direction versus the relative thermal conductivity of the particle for various values of the aspect ratio.

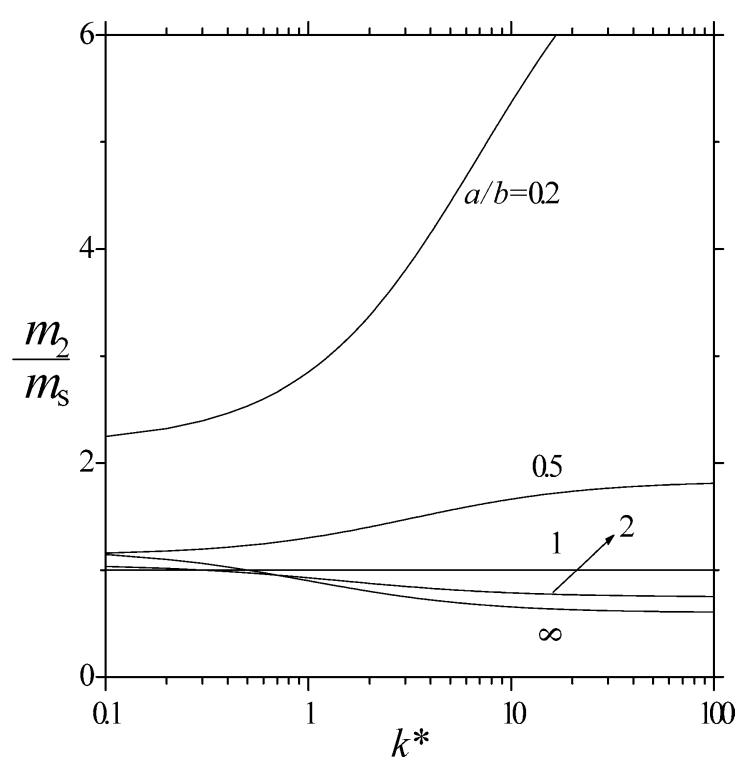

Fig. 5. Plots of the normalized photophoretic mobility of a spheroidal particle in the transverse direction versus the relative thermal conductivity of the particle for various values of the aspect ratio.

thickness with a finite thermal conductivity oriented normal to a light beam has a uniform surface temperature and will not move thermophoretically or photophoretically. For the case of $a / b \rightarrow \infty, m_{1} / m_{s} \rightarrow \infty$, as predicted by Eqs. (47) and (44). For a prolate spheroid with a given aspect ratio, $m_{1} / m_{s}$ is a monotonic increasing function of $k^{*}$, whereas for an oblate spheroid with a fixed value of $a / b$, this normalized mobility is a monotonic decreasing function of $k^{*}$. The reason for this behavior is that the mobility $m_{1}$ decreases with an increase in $k^{*}$ and the sensitivity of this dependence increases with a decrease in $a / b$, as can be seen in Eq. (31). For either a prolate spheroid or an oblate spheroid, an increase in the value of $k^{*}$ in general leads to a greater deviation of the photophoretic mobility from the spherical value $m_{s}$. This deviation can be significant even when the value of $a / b$ does not deviate much from unity. In the limiting case of $k^{*}=0$, $m_{1} / m_{s}=-6 \alpha J_{1}$, and in the other limiting case of $k^{*} \rightarrow \infty$, $m_{1} / m_{s}=-3 \beta J_{1}$, as required by Eq. (31). The behavior in which the curve of $k^{*}=0$ goes up to a nonzero value when $a / b$ approaches zero, unlike all the other curves in Fig. 2a, is physically correct, knowing that a disk of zero thermal conductivity exposed to an axial heat flux will have a singular surface temperature distribution or an infinitely large surface temperature gradient and can undergo the thermophoretic or photophoretic migration. Thus, a curve of small finite value of $k^{*}$ (say, $0<k^{*}<0.2$ ) in Fig. 2 a exhibits a maximum, owing to the competing effects of $a / b$ and $k^{*}$. Note that, for cases of an oblate spheroid with $k^{*}>10$, the value of $m_{1} / m_{s}$ is not a sensitive function of $k^{*}$ and it is equal to $a / b$ approximately, as shown in Fig. 2a.

On the other hand, as illustrated in Figs. 3 and 5, the normalized transverse mobility $m_{2} / m_{S}$ of a photophoretic spheroid increases monotonically with a decrease in its aspect ratio $a / b$ for a constant practical value of $k^{*}$. This trend is caused by the fact that the fraction of the thermal slip of the fluid at the particle surface in the direction of particle's movement (the transverse direction) increases with the decrease of $a / b$. Thus, the spherical approximation underestimates the transverse photophoretic mobility for oblate spheroids and overestimates it for prolate ones. For the special case of $a / b=0, m_{2} / m_{s} \rightarrow \infty$, as given by Eqs. (46) and (44). For the case of $a / b \rightarrow \infty, m_{2} / m_{s}=$ $3\left(2+k^{*}\right) / 5\left(1+k^{*}\right)$, which is consistent with Eq. (48). As a contrast to the tendency of dependence of $m_{1} / m_{s}$ on $k^{*}$, for a prolate spheroid with a specified value of $a / b, m_{2} / m_{s}$ is a monotonically decreasing function of $k^{*}$, while for an oblate spheroid with a fixed aspect ratio, this mobility is a monotonically increasing function of $k^{*}$. This behavior is understandable from the fact that the mobility $m_{2}$ decreases with an increase in $k^{*}$ and the sensitivity of this dependence increases with an increase in $a / b$, as can be found in Eq. (32). Again, for a spheroid of a specified shape, an increase in the value of $k^{*}$ in general results in a greater deviation of the photophoretic mobility from the spherical value $m_{s}$. In the limit of $k^{*}=0, m_{2} / m_{s}=-3 \beta J_{2}$, and in the other limit of $k^{*} \rightarrow \infty, m_{2} / m_{s}=-3(\alpha+1) J_{2} / 2$, as predicted by Eq. (32). Thus, in Fig. 3b, a curve of $k^{*} \leqslant 0.5$ exhibits a trend different from that of $k^{*} \geqslant 1$, owing to the competing effects of $a / b$ and $k^{*}$. For a prolate spheroid with a fixed practical value of $k^{*}$, one always has $m_{1}>m_{s}>m_{2}$, whereas for an oblate spheroid the reverse is true.

\section{Conclusions}

The photophoretic motion of a prolate or oblate spheroidal particle is analyzed and the shape dependence of photophoresis of the particle is investigated in this work. The 
light beam can be applied in an arbitrary direction relative to the axis of revolution of the spheroid. It is assumed that the Knudsen number is small, so that the fluid flow is in the continuum regime and the Knudsen layer at the particle surface is thin in comparison with the linear dimensions of the particle. To solve the conservative equations governing the system in the limit of small Reynolds and Peclet numbers, a thermal slip velocity of fluid and the continuity of temperature and thermal flux at the particle surface are used as the boundary conditions. Explicit expressions for the photophoretic velocity of the spheroidal particle were obtained in Eq. (31) for the longitudinal incident light and in Eq. (32) for the transversal incident light. This photophoretic velocity has shown large variations in magnitude dependent on the shape, relative thermal conductivity, and relative orientation of the particle. Due to the linearity of the system considered, the photophoretic velocity and force of the spheroid exposed to an arbitrarily oriented radiative flux can be obtained by the vectorial addition of the contributions generated from the longitudinal and transverse components of the applied radiative flux, as given by Eqs. (30) and (41). The average photophoretic velocity and force for an ensemble of identical, noninteracting, randomly distributed spheroids can also be calculated from the longitudinal and transversal mobilities of the particle according to Eqs. (42) and (43).

In the previous section, we have presented the results of photophoretic mobility calculated from a simplified model for a completely opaque spheroid whose heat sources are concentrated on the illuminated part of the particle surface. A calculation according to the Lorenz-Mie theory showed bad applicability of this model even for large strongly absorbing spherical particles [29]. Evidently, if we perform a corresponding calculation for a model transparent spheroid with heat sources concentrated completely on the rear half of the particle surface, the same results but with opposite direction (negative photophoresis) will be obtained. Also, for the particular case of the symmetric heat source functions $B_{1}$ and $B_{2}$ (with respect to the planes $z=0$ and $x=0$, respectively), the photophoretic mobility of the spheroid vanishes. Thus, if one uses an energy-absorbing model more consecutive than that defined by Eqs. (37) and (38) for the asymmetry factors $J_{1}$ and $J_{2}$ given by Eqs. (33) and (34) based on the Lorenz-Mie theory, it is expectable that the resulted photophoretic mobility of the spheroid, which can easily be calculated from Eqs. (31) and (32), will be qualitatively similar to but quantitatively smaller than that exhibited in Figs. 2-5.

An examination of Eqs. (1) and (2) reveals that the effect of a finite Knudsen number (say, of order 0.1) is to decrease the gas-kinetic photophoretic mobility of a spherical particle with zero Knudsen number. Although the boundary conditions used in Eqs. (12) and (25) to obtain Eqs. (31) and (32) are strictly valid only for the case of small Knudsen numbers (or large particles), a comparison of the results obtained for a sphere and a cylinder for moderate Knudsen numbers $[8,9,20]$ indicates that the exclusion of effects of frictional slip and temperature jump at the particle surface may not alter the trend of the dependence of photophoresis on particle shape. On the other hand, neglecting these effects is selfconsistent in the analysis of photophoresis. In the model for the continuum regime, the thermal slip at the particle surface given by Eq. (27) (involving the coefficient $C_{S}$ ) is mathematically the zeroth-order (primary) effect in the Knudsen number, as indicated from a comparison between Eq. (1) and Eq. (2). Therefore, the fluid flow around the particle is of the same order of magnitude. However, both the frictional slip (involving $C_{m}$ ) and the velocity slip due to the temperature jump (involving $C_{t}$ ) are the first-order (secondary) effects in the Knudsen number. If one considers the frictional slip and temperature jump in the analysis, it is not consistent because there are some other first-order effects to be taken into account, such as the velocity slip due to the curvature of the particle surface and the contribution of the Knudsen layer to the heat flow. These situations are clear from the systematic asymptotic theory for the Boltzmann equation for a small Knudsen number [13]. Therefore, the closed-form analytical results obtained here, which can be conveniently applied in the calculations for various cases of large particles [24,25], should be a useful contribution to the understanding of the effects of the shape and relative orientation of a particle in photophoresis. The relevant experimental data for the photophoresis of spheroidal particles, which are not available in the literature yet, would be needed to confirm the validity of these results.

\section{Acknowledgment}

Part of this research was supported by the National Science Council of the Republic of China.

\section{Appendix A. Derivation of Eq. (16)}

For conciseness the derivation of Eq. (16) is given here. In bifocal coordinates, Eq. (6), together with Eqs. (7) and (8), takes the form

$$
\begin{gathered}
\frac{\partial}{\partial \tau}\left(\tau^{2}-1\right) \frac{\partial T_{i}}{\partial \tau}+\frac{\partial}{\partial \omega}\left(1-\omega^{2}\right) \frac{\partial T_{i}}{\partial \omega} \\
+\frac{(i-1)\left(\tau^{2}-\omega^{2}\right)}{\left(\tau^{2}-1\right)\left(1-\omega^{2}\right)} \frac{\partial^{2} T_{i}}{\partial \phi^{2}} \\
=-c^{2}\left(\tau^{2}-\omega^{2}\right) g_{i}, \quad i=1,2,
\end{gathered}
$$

where

$$
\begin{aligned}
& g_{1}(\tau, \omega)=\frac{4 \pi \nu \kappa}{\lambda k_{p}} I_{1} B_{1}(\tau, \omega), \\
& g_{2}(\tau, \omega, \phi)=\frac{4 \pi \nu \kappa}{\lambda k_{p}} I_{2} B_{2}(\tau, \omega, \phi) .
\end{aligned}
$$

The solution to Eq. (A.1) can be taken as the sum of the homogeneous and particular solutions, denoted by $T_{i}^{(h)}$ and 
$T_{i}^{(p)}$, respectively. The homogeneous solution is

$T_{i}^{(h)}=\frac{I_{i} c}{k_{p}} \sum_{n=i-1}^{\infty} D_{i n} P_{n}^{i-1}(\omega) P_{n}^{i-1}(\tau) \cos [(i-1) \phi]$,

where $D_{i n}$ are undetermined coefficients.

The particular solution $T_{i}^{(p)}$ can be obtained from a transformation of Eq. (A.1) into an ordinary differential equation. Multiplying Eq. (A.1) through by $P_{n}^{0}(\omega) \mathrm{d} \omega$ and $P_{n}^{1}(\omega) \cos \phi \mathrm{d} \omega \mathrm{d} \phi$ for the cases of $i$ equal to 1 and 2, respectively, and integrating from -1 to 1 for $\omega$ and from 0 to $2 \pi$ for $\phi$ lead to

$\frac{\partial}{\partial \tau}\left(\tau^{2}-1\right) \frac{\partial R_{i n}}{\partial \tau}-\left[n(n+1)-\frac{i-1}{\tau^{2}-1}\right] R_{i n}=-h_{i n}$,

where

$$
\begin{aligned}
R_{1 n}(\tau)= & \frac{k_{p}}{I_{1} c} \int_{-1}^{1} T_{1}^{(p)} P_{n}^{0}(\omega) \mathrm{d} \omega, \\
h_{1 n}(\tau)= & \frac{k_{p} c}{I_{1}} \int_{-1}^{1}\left(\tau^{2}-\omega^{2}\right) g_{1}(\tau, \omega) P_{n}^{0}(\omega) \mathrm{d} \omega, \\
R_{2 n}(\tau)= & \frac{k_{p}}{I_{2} c \pi} \int_{0}^{2 \pi} \int_{-1}^{1} T_{2}^{(p)} P_{n}^{1}(\omega) \cos \phi \mathrm{d} \omega \mathrm{d} \phi, \\
h_{2 n}(\tau)= & \frac{k_{p} c}{I_{2} \pi} \int_{0}^{2 \pi} \int_{-1}^{1}\left(\tau^{2}-\omega^{2}\right) g_{2}(\tau, \omega, \phi) \\
& \times P_{n}^{1}(\omega) \cos \phi \mathrm{d} \omega \mathrm{d} \phi .
\end{aligned}
$$

Obviously, Eqs. (A.6) and (A.8) lead to

$T_{i}^{(p)}=\frac{I_{i} c}{k_{p}} \sum_{n=i-1}^{\infty} \frac{R_{i n}(\tau) P_{n}^{i-1}(\omega) \cos [(i-1) \phi]}{\int_{-1}^{1}\left[P_{n}^{i}(\omega)\right]^{2} \mathrm{~d} \omega}$.

The solution to Eq. (A.5) can be obtained by the method of variation of parameters, with the result give by Eq. (17).

The combination of Eqs. (A.4) and (A.10) incorporated with Eq. (5) results in Eq. (16). The substitution of Eqs. (A.2) and (A.3) into Eqs. (A.7) and (A.9) yields Eqs. (19) and (20).

\section{Appendix B. Nomenclature}

$a$ half-length of a spheroid along its axis of revolution, $\mathrm{m}$

$B_{1}, B_{2}$ dimensionless electric field distribution functions defined by Eqs. (7) and (8)

$b \quad$ maximum cross-sectional radius of a spheroid, $\mathrm{m}$

$C_{s} \quad$ thermal slip coefficient

$C_{i n}, D_{i n}$ coefficients in Eqs. (15) and (16) given by Eqs. (21) and (22) $c \quad$ equal to $\left(a^{2}-b^{2}\right)^{1 / 2}$ if $b<a$ and $\left(b^{2}-a^{2}\right)^{1 / 2}$ if $b>a, \mathrm{~m}$

$\mathbf{e}_{x}, \mathbf{e}_{z} \quad$ unit vectors in the $x$ and $z$ directions

I intensity of the incident light beam, $\mathrm{W} \mathrm{m}^{-2}$

$I_{1}, I_{2}$ longitudinal and transversal components of $\mathbf{I}$ relative to a spheroid, $\mathrm{W} \mathrm{m}^{-2}$

$J, J_{1}, J_{2}$ the photophoretic asymmetry factors of the particle defined by Eqs. (33) and (34)

$k$ thermal conductivity of the fluid, $\mathrm{W} \mathrm{m}^{-1} \mathrm{~K}^{-1}$

$k_{p} \quad$ thermal conductivity of the particle, $\mathrm{W} \mathrm{m}^{-1} \mathrm{~K}^{-1}$

$k^{*} \quad$ equal to $k_{p} / k$

$l \quad$ mean free path of the gas molecules, $m$

$m_{1}, m_{2}$ longitudinal and transversal components of the photophoretic mobility of a spheroid, $\mathrm{m}^{3} \mathrm{~J}^{-1}$

$m_{s} \quad$ photophoretic mobility of a sphere given by Eq. (44), $\mathrm{m}^{3} \mathrm{~J}^{-1}$

n unit normal vector at particle surface pointing into the fluid

$P_{1}^{m}, Q_{1}^{m}$ associated Legendre functions of the first and second kinds

$R_{\text {in }}(\tau)$ functions of $\tau$ defined by Eqs. (16) and (17)

$\mathbf{r}$ position vector relative to the particle center, $\mathrm{m}$

$S_{p} \quad$ particle surface

$T$ temperature distribution in the fluid, $\mathrm{K}$

$T_{0} \quad$ temperature of the isothermal bulk fluid, $\mathrm{K}$

$T_{p} \quad$ temperature distribution inside the particle, $\mathrm{K}$

U photophoretic velocity, $\mathrm{m} \mathrm{s}^{-1}$

$U_{1}, U_{2}$ longitudinal and transversal components of $\mathbf{U}$, $\mathrm{m} \mathrm{s}^{-1}$

$\mathbf{v} \quad$ fluid velocity field, $\mathrm{m} \mathrm{s}^{-1}$

$\mathbf{v}_{S} \quad$ slip velocity at the particle surface, $\mathrm{m} \mathrm{s}^{-1}$

$x, y, z$ rectangular coordinates, $\mathrm{m}$

\section{Greek letters}

$\alpha, \beta \quad$ functions of $\tau_{0}$ or $a / b$ defined by Eqs. (35) and (36)

$\eta \quad$ fluid viscosity, $\mathrm{kg} \mathrm{m}^{-1} \mathrm{~s}^{-1}$

$\lambda$ the wavelength of the incident light beam, $m$

$\xi, \varphi, \phi$ bifocal coordinates

$\rho \quad$ fluid density, $\mathrm{kg} \mathrm{m}^{-3}$

$\tau \quad$ equal to $\cosh \xi$ if $b<a$ and $i \sinh \xi$ if $b>a$

$\tau_{0} \quad$ equal to $\left[1-(b / a)^{2}\right]^{-1 / 2}$

$\nu, \kappa \quad$ real and imaginary parts of the complex refractive index $\bar{N}$ of the particle

$\omega \quad$ equal to $\cos \varphi$

\section{References}

[1] C. Orr, E.Y.H. Keng, J. Atmos. Sci. 21 (1964) 475-478.

[2] O. Preining, in: C.N. Davies (Ed.), Aerosol Science, Academic Press, New York, 1966, pp. 111-135.

[3] J.C. Maxwell, Philos. Trans. R. Soc. London 170 (1879) 231-256.

[4] E.H. Kennard, Kinetic Theory of Gases, McGraw-Hill, New York, 1938, pp. 291-337.

[5] S. Arnold, M.J. Lewittes, J. Appl. Phys. 53 (1982) 5314-5319.

[6] V. Chernyak, S. Beresnev, J. Aerosol Sci. 24 (1993) 857-866. 
[7] M. Kerker, D.D. Cooke, J. Opt. Soc. Am. 72 (1982) 1267-1272.

[8] D.W. Mackowski, Int. J. Heat Mass Transfer 32 (1989) 843-854.

[9] L.D. Reed, J. Aerosol Sci. 8 (1977) 123-131.

[10] Yu.I. Yalamov, V.B. Kutukov, E.R. Shchukin, J. Colloid Interface Sci. 57 (1976) 564-571.

[11] L. Talbot, R.K. Cheng, R.W. Schefer, D.R. Willis, J. Fluid Mech. 101 (1980) 737-758.

[12] C. Cercignani, Rarefied Gas Dynamics: From Basic Concepts to Actual Calculations, Cambridge Univ. Press, Cambridge, UK, 2000.

[13] Y. Sone, Kinetic Theory and Fluid Dynamics, Birkhäuser, Boston, 2002.

[14] B.E. Dahneke, Aerosol Sci. 4 (1973) 139-145.

[15] M.L. Laucks, G. Roll, G. Schweiger, E.J. Davis, J. Aerosol Sci. 31 (2000) 307-319.

[16] H. Rohatschek, W. Zulehner, J. Colloid Interface Sci. 108 (1985) 457.

[17] W. Zulehner, H. Rohatschek, J. Colloid Interface Sci. 138 (1990) 555.

[18] W. Zulehner, H. Rohatschek, J. Aerosol Sci. 26 (1995) 201.
[19] D.W. Mackowski, J. Colloid Interface Sci. 140 (1990) 138.

[20] H.J. Keh, H.J. Tu, Colloids Surf. A 176 (2001) 213-223.

[21] W.M. Greene, R.E. Spjut, E. Bar-Ziv, A.F. Sarofim, J.P. Longwell, J. Opt. Soc. Am. B 2 (1985) 998-1004.

[22] A.F.M. Akhtaruzzaman, S.P. Lin, J. Colloid Interface Sci. 61 (1977) $170-182$.

[23] P. Moon, D.E. Spencer, Field Theory Handbook, Springer-Verlag, Berlin, 1961.

[24] K.H. Leong, J. Aerosol Sci. 15 (1984) 511-517.

[25] M.M.R. Williams, J. Phys. D 19 (1986) 1631-1642.

[26] J. Happel, H. Brenner, Low Reynolds Number Hydrodynamics, Martinus Nijhoff, 1983.

[27] C.N. Davies, J. Aerosol Sci. 10 (1979) 477-513.

[28] M.C. Fair, J.L. Anderson, J. Colloid Interface Sci. 127 (1989) 388400.

[29] S.A. Beresnev, L.B. Kochneva, Atmos. Oceanic Opt. 16 (2003) 119126. 